\title{
Predicting survival outcomes using subsets of significant genes in prognostic marker studies with microarrays Shigeyuki Matsui*1,2
}

\author{
Address: ${ }^{1}$ Department of Pharmacoepidemiology, School of Public Health, Kyoto University, Yoshida Konoe-cho, Sakyo-ku, Kyoto 606-8501, \\ Japan and ${ }^{2}$ Translational Research Informatics Center, Foundation for Biomedical Research and Innovation, Minatojima-minami-machi, Chuo- \\ $\mathrm{ku}$, Kobe 650-0047, Japan \\ Email: Shigeyuki Matsui* - matsui@pbh.med.kyoto-u.ac.jp \\ * Corresponding author
}

Published: 20 March 2006

BMC Bioinformatics 2006, 7:156 doi:10.1186/147/-2105-7-156
Received: 0I September 2005

Accepted: 20 March 2006

This article is available from: http://www.biomedcentral.com/I47/-2105/7//56

(C) 2006 Matsui; licensee BioMed Central Ltd.

This is an Open Access article distributed under the terms of the Creative Commons Attribution License (http://creativecommons.org/licenses/by/2.0), which permits unrestricted use, distribution, and reproduction in any medium, provided the original work is properly cited.

\begin{abstract}
Background: Genetic markers hold great promise for refining our ability to establish precise prognostic prediction for diseases. The development of comprehensive gene expression microarray technology has allowed the selection of relevant marker genes from a large pool of candidate genes in early-phased, developmental prognostic marker studies. The primary analytical task in such studies is to select a small fraction of relevant genes, typically from a list of significant genes, for further investigation in subsequent studies.
\end{abstract}

Results: We develop a methodology for predicting survival outcomes using subsets of significant genes in prognostic marker studies with microarrays. Key components in this methodology include building prediction models, assessing predictive performance of prediction models, and assessing significance of prediction results. As particular specifications, we assume Cox proportional hazard models with a compound covariate. For assessing predictive accuracy, we propose to use the cross-validated log partial likelihood. To assess significance of prediction results, we apply permutation procedures in cross-validated prediction. As an additional key component peculiar to prognostic prediction, we also consider incorporation of standard prognostic factors. The methodology is evaluated using both simulated and real data.

Conclusion: The developed methodology for prognostic prediction using a subset of significant genes can provide new insights based on predictive capability, possibly incorporating standard prognostic factors, in selecting a fraction of relevant genes for subsequent studies.

\section{Background}

Genetic markers hold great promise for refining our ability to establish precise prognostic prediction for diseases. The development of comprehensive, gene expression microarray technology has allowed the selection of relevant marker genes from a large pool of candidate genes in early-phased, developmental prognostic marker studies for various cancers including diffuse large B-cell lym- phoma [1], follicular lymphoma [2], acute myeloid leukemia [3], lung adenocarcinoma [4], and metastatic kidney cancer [5]. The selected genes will be further investigated in subsequent studies using technically simpler, but more reliable assays such as multiplexed quantitative reversetranscriptase polymerase chain reaction (RT-PCR) in formalin-fixed, paraffin-embedded tissue sections for routine clinical use $[6,7]$. Accordingly, the primary task in 
early-phased prognostic marker studies with microarrays would be to select a small fraction of relevant genes for subsequent studies. To this end, multiple testing to identify genes associated with prognosis is typically adopted as primary analysis, which may provide a list of significant genes.

Prediction analysis using subsets of significant genes may supplement the primary analysis. It can provide information regarding predictive capability for subsets of significant genes. More importantly, provided that appropriate measures of predictive accuracy for survival outcomes are established, it may indicate another 'cut-off for a list of significant genes on the basis of predictive accuracy through gene filtering other than the criteria to control false positives in multiple testing. Despites many methods for prediction analysis of survival outcomes proposed in bioinformatics and biostatistics literature, including application of partial least squares [8-10] and ridge regression $[11,12]$, most methods intend to use the full set of genes for prediction without regard to the primary analysis.

In this article, we develop a methodology for predicting survival outcomes using subsets of significant genes. Key components in this methodology include building prediction models, assessing predictive performance of prediction models, and assessing significance of prediction results. Here, given the first two components, we can perform gene filtering. For each component, we consider particular specifications or procedures to illustrate the methodology. In building prediction models, we assume Cox proportional hazard models with a compound covariate $[13,14]$. In assessing performance of prediction models, measures of explained variation for Cox regression models [15-17] may not aim to measure the performance of prediction models on future patients, i.e., predictive accuracy. We propose to use the cross-validated log partial likelihood $[18,19]$ to measure predictive accuracy. To assess significance of prediction results, we apply the permutation procedures in cross-validated prediction proposed by Radmacher et al. [14]. As an additional key component peculiar to prognostic prediction, we also consider incorporation of standard prognostic factors, because it is important to determine whether a new genetic marker adds prognostic information to that already contained in the more established prognostic factors [20]. The performance of the methodology will be evaluated using simulated data and real data from a lymphoma study.

\section{Results \\ Gene filtering}

The simplest approach of gene filtering is based on the marginal association between each gene expression and survival time [1-5]. For patient $i$ in the training set, let $h_{i}(t)$ be the hazard function and $x_{j, i}$ be the expression level for gene $j$. For gene $j$, we assume the univariate Cox regression model,

$$
h_{i}(t)=h_{j, 0}(t) \exp \left(\beta_{j} x_{j, i}\right)
$$

where $h_{j, 0}(t)$ is the baseline hazard function and $\beta_{j}$ is a parameter. Gene filtering is based on a test of hypothesis $\beta_{j}=0$ (e.g., a score or Wald test [21]). Genes are typically ranked on the basis of the value of absolute standardized test statistic. Gene filtering can be based on the number of genes $[4]$ or a $P$-value cut-off $[1,2,5]$. A standardized score or Wald test statistic for testing hypothesis $\beta_{j}=0$ is asymptotically normal with unit variance and mean equal to $D^{1 /}$ ${ }^{2} \beta_{j} \sigma_{j}$ where $\sigma_{j}^{2}$ is the variance of expression levels across patients for gene $j$ and $D$ is the expected number of events [22]. The gene filtering is thus based on the hazard ratio associated with a change of standard deviation in gene expression for a given number of events.

\section{Prediction model}

For the set of $K$ selected genes $\left(j_{1}, \ldots, j_{K}\right)$, the compound covariate for patient $i$ is defined as

$c_{i}=\sum_{k=1}^{K} z_{j_{k}} x_{j_{k}, i}$

where $z_{j_{k}}$ is the standardized test statistic obtained in the gene filtering for the selected gene $j_{k}(k=1, \ldots, K)$. The definition of the compound covariates weights by means of standardized test statistics has been suggested for generalized linear models in Radmacher et al. [14]. This weighting policy reflects the criterion in the gene filtering step. Another possible policy is to use an estimate of $\beta_{j}$, in stead of $z_{j}$, as the weight for gene $j$ (e.g., Beer et al. [4]). Our weighting policy gives higher weight to genes with larger variance, which would yield a more robust predictor for subsequent validation studies because the expression profiles for genes with larger variance would be more reproducible.

The compound covariate can be regarded as a prognostic index; patients with large values of the compound covariate may have poor prognosis. We assume the following Cox model to relate the compound covariate to the survival time,

$h_{i}(t)=h_{0}(t) \exp \left(\psi c_{i}\right)$ 
where $h_{0}(t)$ is the baseline hazard function and $\psi$ is a parameter. The compound covariate for a new patient with the vector of expression level $\left(x_{j_{1}}^{*}, \ldots, x_{j_{k}}^{*}\right)$ for the selected genes can be calculated by replacing $x_{j_{k}, i}$ with $x_{j_{k}}^{*}$ in $(2)$, which is used for the prediction of survival time for that patient.

\section{Predictive accuracy}

We use the cross-validated log partial likelihood $[18,19]$ to measure predictive accuracy of Cox models. Specifically, the average $M$-fold cross-validated log partial likelihood is given by

$$
A C V L=-\frac{1}{M} \sum_{m=1}^{M} l_{m}\left(\psi_{(-m)}\right)
$$

where $l_{m}(\psi)=l_{T}(\psi)-l_{(-m)}(\psi)$ is the difference between the partial log likelihood for the entire training set and that with the $m$ th group of patients excluded as the test set, and $\hat{\psi}_{(-m)}$ is the value of $\psi$ that maximizes $l_{(-m)}(\psi)$ for $m=1$, ..., $M$. As to the number of cross-validation groups, $M=10$ or 5 are reasonable choices especially for computationally burdensome analyses for large samples [23]. A low value of ACVL corresponds to high predictive accuracy. ACVL reduces to the prediction residual error of sum of square (PRESS) in normal linear models [18].

When using $M$-fold cross-validation, it is critical that all aspects of model building including gene filtering are reperformed for each of $M$ rounds of cross-validation to avoid selection bias $[24,25]$. If we choose the cut-offs in gene filtering that minimizes $A C V L$, an independent validation set would be needed to have unbiased estimate of predictive accuracy because of the optimization process in model building for the training set. Matsui [26] demonstrated that the bias due to the optimization can be substantial in a class prediction problem from gene expression profiling using 6,000 genes for 48 bladder tumors.

A limitation of $A V C L$ is that it is difficult to interpret for non-statisticians. Some graphical displays may be helpful to interpret the results. If an independent validation set is available, a simple way is to divide the validation set into some groups based on the value of the prognostic index and compare survival curves between groups using a logrank test. The same type of assessment can also be performed for cross-validated test sets from the training set, but a usual log-rank test is not valid because the groups are not pre-specified independently of the survival time. A permutation procedure which permutates survival time to expression profile is available to have a correct $P$-value $[14,5]$. In this procedure, the same cross-validated model building process, with some optimization process such as choosing optimal cut-off based on ACVL, if any, is performed to permutated data to obtain a null distribution of the log-rank statistic. This permutation procedure can also be useful for assessing the statistical significance of (the minimized) $A C V L$, in which one may obtain a null distribution of (the minimized) ACVL.

\section{Adjustment for prognostic factors}

Let $u_{i}$ be a vector of prognostic factors for patient $i$. For gene $j$, we assume the Cox model, instead of (1),

$$
h_{i}(t)=h_{j, 0}(t) \exp \left(\eta_{j}^{\prime} u_{i}+\beta_{j} x_{j, i}\right)
$$

where $\eta_{j}$ is a vector of parameters. Gene filtering is based on a test of hypothesis $\beta_{j}=0$ in model (4). This is to select genes after adjustment for the prognostic factors. For the set of $K$ selected genes $\left(j_{1}, \ldots, j_{K}\right)$, we calculate the compound covariate $c_{i}$ in (2) using a standardized test statistic for the hypothesis $\beta_{j_{k}}=0$. Then we assume the Cox model, instead of (3),

$h_{i}(t)=h_{0}(t) \exp \left(\gamma u_{i}+\psi c_{i}\right)$

where $\gamma$ and $\psi$ are parameters. We assess the predictive accuracy based on ACVL for model (5). The prediction is based on the prognostic index, $\hat{\gamma}^{\prime} u_{i}+\hat{\psi} c_{i}$ where $\hat{\gamma}$ and $\hat{\psi}$ are estimates of $\gamma$ and $\psi$, respectively, obtained from the training set.

Analyses should test whether new systems add predictiveness once outcome is adjusted for the effect of standard prognostic factors [20]. For the validation set, a graphical display similar to that described in the previous section may be drawn for each stratum by prognostic factors and compare survival curves using a log-rank test for each stratum or a stratified log-rank test. For cross-validated test sets, a stratum-adjusted permutation procedure would be useful, in which the observed value of the log-rank statistic or (the minimized) ACVL are referred to their null distribution obtained by permutating survival time to expression profile within each stratum.

\section{Simulated data}

In this section, we assessed adequacy of choosing the cutoffs in gene filtering for the training set based on ACVL for the Cox model (3) through a small simulation study. We simulated data on 2,000 genes for 100 patients. Of the 2,000 genes, 50 genes were configured to be informative, 
i.e., these genes are associated with survival time. For informative genes, the distribution of expression was normal with mean 0 and standard deviation 1 (supposing a standardized expression data across patients for each gene). We considered exchangeable correlation matrices with correlation coefficient $\rho$ of 0.2 or 0.7 . In addition, we considered the correlation matrix obtained from the data from the lymphoma study by Rosenwald et al. [1] for the top 50 genes in the gene filtering with model (1). The range of correlation was -0.53 to 0.98 . The informative genes were associated with survival time via a multivariate proportional hazard model,

\section{$\lambda_{0}(t) \exp \left(\beta^{\prime} x\right)$}

where $\lambda_{0}(t)$ denotes the baseline hazard function, $\beta$ a vector of regression parameters, and $x$ a vector of gene expression for the 50 informative genes. We assumed a constant baseline hazard. We set the value of parameters to mimic the lymphoma data; the baseline hazard was set equal to 0.13 (/year) and all the elements of $\beta$ to $0.5(=\log (1.65))$, corresponding to a 1.65-fold in the hazard of failing with a standard deviation increase in gene expression. Note that the range of the absolute value of the estimate of $\beta$ for the top 50 genes obtained from a standardized lymphoma data was 0.39 to 0.60 . For parameter $\beta$, we also considered an estimate of $\beta$ from the top 50 genes in the lymphoma data, which were mixture of positive and negative values, but we obtained similar results. For non-informative genes, the distribution of expression was normal with mean 0 and variance-covariance equal to the identity matrix. For each of simulated data, we calculated ACVL with $M=5$ for the Cox model (3) with the compound covariate for several values of $K$. For $K \leq 20$, we also calculated $A C V L$ for a multiplicative model of the form of equation (6) that included as covariates the $K$ genes selected during the gene filtering step. Note that using the full set of $K$ genes with $K>20$ gave noninvertible covariance matrices in maximizing the partial likelihood, which is an inherent limitation of fitting multivariate Cox models with a number of predictors. We considered a constant censoring rate of 0.1 (/year), again, to mimic the lymphoma data. Table 1 shows the averaged ACVL for each of several values of $K$ across 100 simulations. The ACVL for the model (3) was minimized in expectation when the cut-off in terms of the number of selected genes $(K)$ was set equal to the number of informative genes, 50. The ACVL for the model (3) was smaller than that for the multivariate Cox model $(6)$ for the top $K(\leq 20)$ genes.

\section{Lymphoma data}

We illustrated the developed methodology using the data from Rosenwald et al. [1] for diffuse large B-Cell lymphoma http://lmpp.nih.gov/DLBCL/. Briefly, this study collected gene expression data from cDNA microarrays using pretreatment biopsy specimens and clinical data for 240 diffuse large B-Cell lymphoma patients. Clinical data included the International Prognostic Index (IPI) Score [27], which is a composite score reflecting age, tumor stage, performance status, lactate dehydrogenase level, and the number of sites of extranodal disease, before treatment as a prognostic factor. 7399 microarray features were subject to analysis for predicting the survival time after treatment. In the prediction analysis, the patients were randomly divided into two groups: the training set comprised 160 patients and the validation set comprised 80 patients. The number of events (the median survival in year) was 88 (3.9) for the training set and 50 (3.6) for the validation set.

Before demonstrating our methods, we assessed the prognostic value of IPI Score. In the dataset, IPI score had three levels, low, medium, and high in terms of the risk of death. Because only 10 out of 80 patients were in the high IPI risk stratum in the validation set, we combined the medium and high IPI risk stratum into one stratum and referred to it as the high IPI risk stratum. Figure 1 shows estimated survival distributions for the low IPI risk stratum (27 patients) and the high IPI risk stratum (46 patients) in the validation set (IPI Score was missing for 7 patients), which indicates a substantial prognostic capability of IPI Score (the P-value of a log-rank test was 0.0076).

First we performed prediction only using gene expression data. We performed 5-fold cross-validation in building prediction models with the compound covariate for the training set. For each fold of cross-validation, we performed gene filtering from scratch to select the top $K$ genes in terms of the significance level for a Wald test of $\beta_{j}=0$ for model (1) and then fitted the model (3) with the compound covariate (2) from the top $K$ genes. We chose the optimal number of genes based on ACVL for model (3). Figure 2 shows $A C V L$ for a range of $K$, indicating that $A C V L$ is minimized when $K=75$. The $P$-value for the minimized $A C V L$ obtained by the permutation procedure $(2,000$ permutations) for the training set was less than 0.0005 , which was highly significant.

To compare the predictive capability of the compound covariate from the 75 selected genes with that of IPI Score for the validation set, we divided the validation set into two groups based on the value of compound covariate from the 75 selected genes using the division ratio of 27: 46 , which is identical with the ratio when the patients in the validation set is divided based on the two-leveled IPI Score mentioned above. Figure 3 shows estimated survival distributions for the two groups in the validation set without and with stratification by IPI Score. The 75 selected genes had a predictive capability by themselves (the $P$ - 


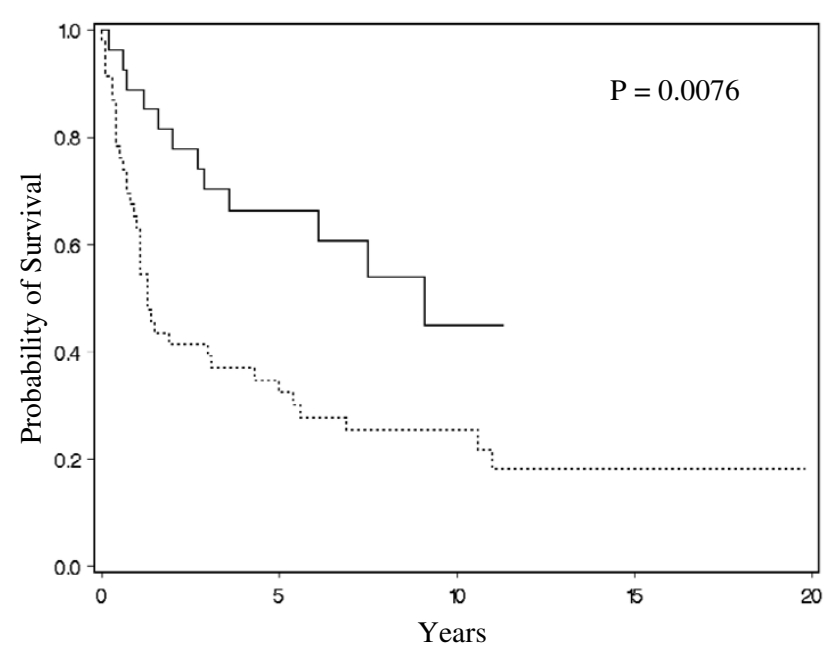

Figure I

Kaplan-Meier estimates of survival for the low IPI stratum (solid curves) and the high IPI stratum (dashed curves) in the validation set. The $P$-value pertains to a two-sided log-rank test.

value of a log-rank test was 0.0607), but it was rather weak, especially, for the low IPI risk stratum (the P-value of a log-rank test was 0.5530 for this stratum and the $P$ value of a stratified log-rank test was 0.0941).

Next, we performed gene filtering after adjustment for IPI Score and built prediction models using both IPI Score

Table I: The averaged ACVL for several values of $K$ across 100 simulations. Of the $\mathbf{2 , 0 0 0}$ genes, 50 genes were configured to be informative. The number of patients was 100.

\begin{tabular}{|c|c|c|c|c|c|c|}
\hline \multirow[b]{4}{*}{$K$} & \multicolumn{6}{|c|}{ Correlation matrix } \\
\hline & \multicolumn{4}{|c|}{ Exchangeable } & \multicolumn{2}{|c|}{ Lymphoma data* } \\
\hline & \multicolumn{2}{|c|}{$\rho=0.2$} & \multicolumn{2}{|c|}{$\rho=0.7$} & \multirow[b]{2}{*}{$\mathrm{CC}$} & \multirow[b]{2}{*}{$\mathrm{MC}$} \\
\hline & $\mathrm{CC}^{+}$ & $M C \ddagger$ & $\mathrm{CC}$ & $M C$ & & \\
\hline 1 & 41.2 & 41.2 & 51.6 & 51.6 & 48.2 & 48.2 \\
\hline 5 & 33.3 & 34.1 & 46.7 & 48.2 & 45.6 & 46.4 \\
\hline 10 & 29.3 & 33.2 & 43.3 & 46.6 & 45.2 & 48.2 \\
\hline 20 & 24.9 & 49.0 & 38.4 & 62.1 & 45.0 & 57.4 \\
\hline 50 & 17.3 & - & 34.6 & - & 41.7 & - \\
\hline 75 & 21.3 & - & 40.1 & - & 42.3 & - \\
\hline 100 & 25.3 & - & 44.0 & - & 43.2 & - \\
\hline 200 & 36.0 & - & 53.3 & - & 47.0 & - \\
\hline 500 & 50.6 & - & 63.6 & - & 53.9 & - \\
\hline 1000 & 56.6 & - & 70.3 & - & 58.8 & - \\
\hline
\end{tabular}

*The correlation matrix obtained from the lymphoma data for the top 50 genes in the gene filtering with model (I).

tCox model with the compound covariate (model (3))

¥Multivariate Cox model (model $(6))$ for the top $K(\leq 20)$ genes

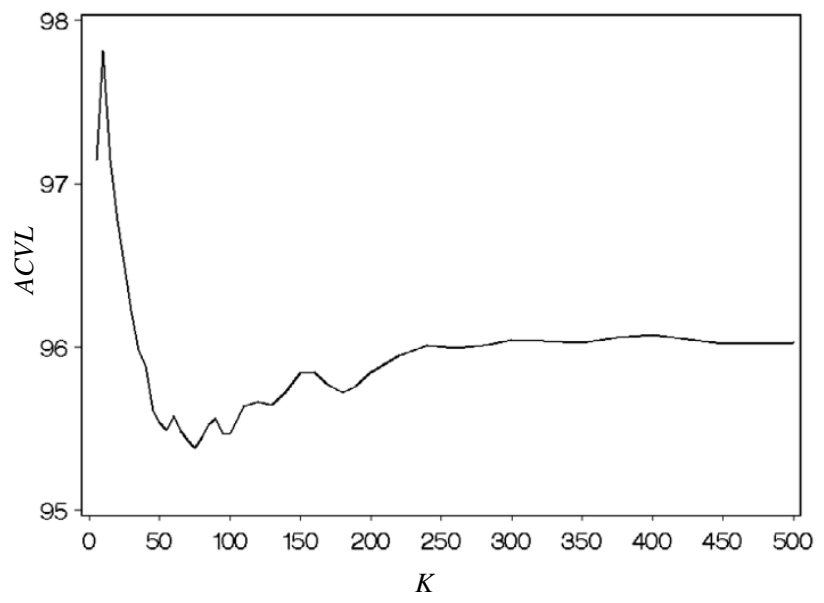

Figure 2

Plot of $A C V L$ as $K$ is varied. The $A C V L$ is minimized when $K=$ 75.

and gene expression data. This analysis would be warranted in the presence of substantial predictive capability of IPI Score as indicated by Figure 1. Again, we performed 5 -fold cross-validation for the training set. Gene filtering was based on a Wald test of $\beta_{j}=0$ for model (4) with $u_{i}$ for IPI Score $\left(u_{i}=1\right.$ if IPI Score is high, and $u_{i}=0$ otherwise). Figure 4 shows $A C V L$ for model (5) as $K$ is varied, indicating that $A C V L$ is minimized when $K=85$. The $P$-value from the stratum-adjusted permutation procedure $(2,000$ permutations) to assess statistical significance of the minimized ACVL after adjustment for IPI Score was 0.024, which was still significant at the significance level of 0.05 . This means that gene expression data have additional prognostic information independent of IPI Score.

For the validation set, Figure 5 shows estimated survival distributions between the two groups based on the prognostic index without and with stratification by IPI Score. The predictive capability of both IPI Score and the 85 selected genes was substantial (the $P$-value of a log-rank test was 0.0063 ). The predictive capability for the IPI low risk stratum was improved (the $P$-value of a log-rank test was 0.0945 for this stratum and the $P$-value of a stratified log-rank test was 0.0293).

Table 2 summarizes the selected genes by gene-expression signatures. 43 genes were selected from both the gene filtering with no adjustment for IPI Score and that with the adjustment (Group I). 32 genes were selected from the gene filtering with no adjustment, but not selected from that with adjustment (Group II). 42 genes were not selected from the gene filtering with no adjustment, but selected from that with adjustment (Group III). It is interesting that Group III contains 7 genes from major histo- 
All patients

(A)

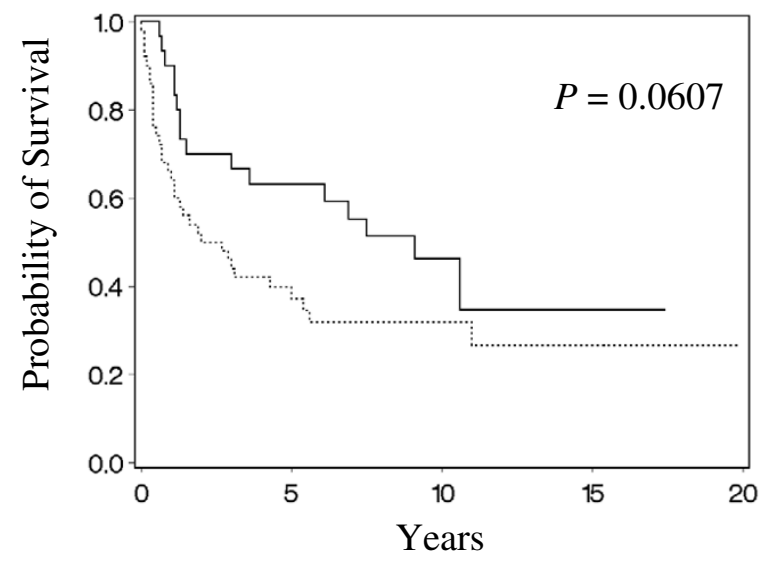

Low IPI risk stratum

(B)

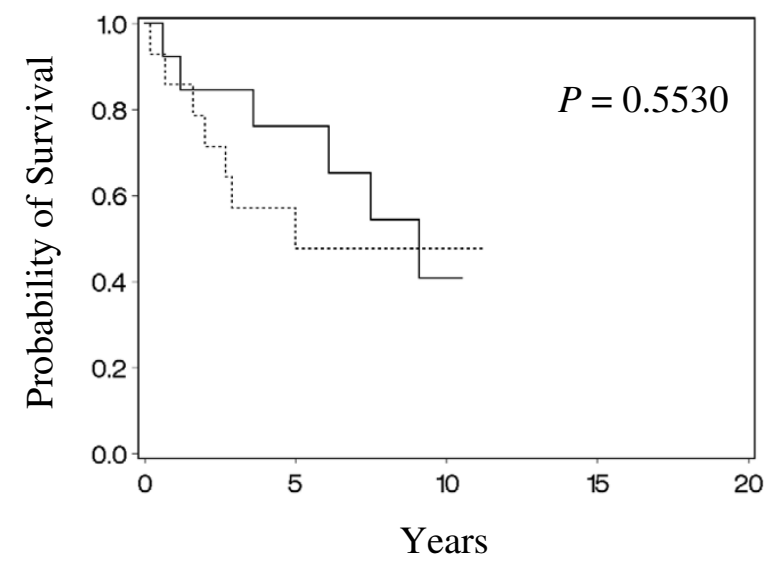

High IPI risk stratum

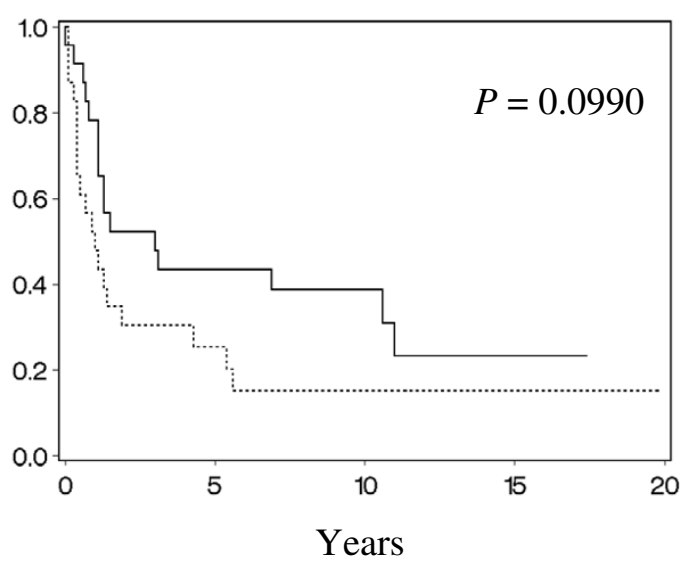

\section{Figure 3}

Kaplan-Meier estimates of survival for the two groups based on the compound covariate (CC) (the low CC group (solid curves) and the high CC group (dashed curves) for all patients (Panel A) and for each stratum by IPI Score (Panel B) in the validation set. The $P$-value in graph pertains to a two-sided log-rank test. The $P$-value for a stratified log-rank test is $0.094 \mathrm{I}$.

compatibility complex (MHC) class II, while Group II contains only one gene from this gene-expression signature. The number of differential expressed genes evaluated by a two-sample $t$-test to compare expressions between the two levels of IPI Score at the significance level of 0.05 was $9(21 \%), 16(50 \%)$, and $0(0 \%)$, for Group I, II, and III, respectively. Group III has a potential to be genetic markers independent of IPI Score.

\section{Discussion}

In this article, we have developed a methodology for predicting survival outcomes using subsets of significant genes in early-phased, developmental prognostic marker studies with DNA microarrays. Key components in this methodology include development of prediction models, assessment of predictive capability of prediction models, and assessment of significance of prediction results. To illustrate the methodology, we introduced a particular prediction model, Cox regression models with a compound covariate, and a particular measure of predictive accuracy, ACVL. Although adequacy of them was indicated through their application to simulated data and real data, further studies to evaluate their performance through comparison with other specifications or methods would be warranted.

With respect to specification of prediction model, Bair and Tibshirani [28] recently developed a semi-supervised method that adopted principal components analysis for developing a compound index using subsets of significant 


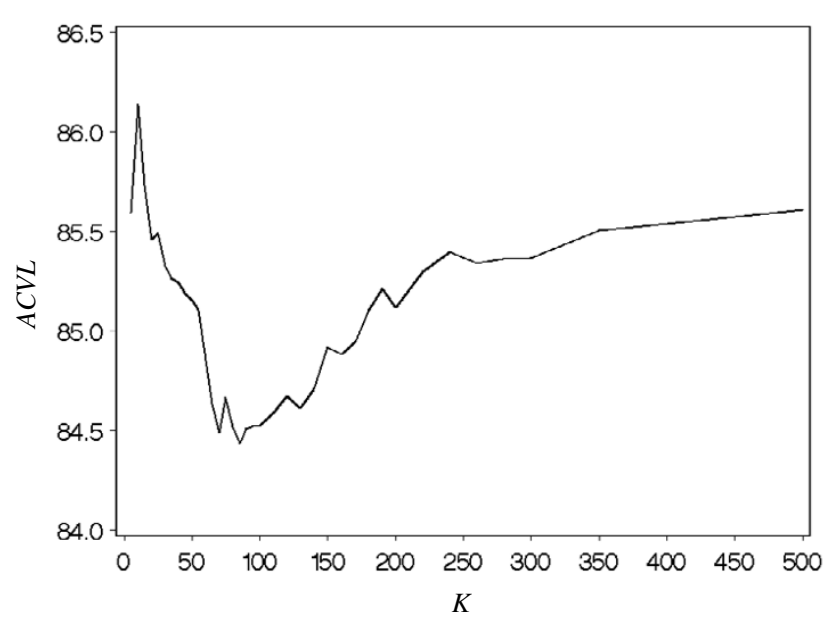

Figure 4

Plot of $A C V L$ as $K$ is varied after adjustment for IPI Score. The $A C V L$ is minimized when $K=85$.

genes from the supervised, univariate Cox regression analysis with model (1). They used the first principal component, in stead of the compound covariate $c_{i}$ in (2), as a single covariate in the Cox model (3). The semi-supervised method using subsets of significant genes performed well compared with various methods using a combination of all of the genes for some cancer datasets and simulated datasets. More recently, Li and Gui [10] considered the application of partial least square and proposed multivariate Cox regression models using the first few orthogonal compound covariates for a full set of genes. The use of the second or higher orthogonal compound covariates proposed by Li and Gui, in addition to the first compound covariate like $c_{i}$ in (2), for subsets of significant genes has the potential to improve predictive accuracy. A comparison study for several prediction methods including those described above using subsets of significant genes is ongoing and the result will appear in a future report. Our method with the compound covariate $c_{i}$ in (2) and the method proposed by Li and Gui using subsets of significant genes are expected to perform well because they are purely supervised. One potential drawback for applying principal components and partial least squares in practice is that they need a complete expression dataset with no missing values for the set of $K$ selected genes. Because there is generally a large number of missing values in the dataset, a complete case analysis [29] will entail a substantial efficiency loss. As such, these methods may necessitate a data imputation step prior to model fitting. Meanwhile, the univariate standardized test statistics as the weights in compound covariate $c_{i}$ in (2) can be calculated using all the observed expression levels for the set of $K$ selected genes, i.e., an available data analysis [29] can be performed.

As to measure of predictive accuracy for survival outcomes, methods based on the time-dependent ROC curves and area under the curves [30] can be useful for microarray data [10].

The significance of integrating gene expression profiling into prognostic prediction studies is to improve the predictive capability attainable only using standard prognostic factors. However, it is rare that prognostic factors are incorporated in the prediction analysis using gene expression data in the literature. As an additional key component of our methodology, we considered selection of relevant genes with the adjustment for prognostic factors. The selected genes have the potential to be genetic markers unrelated to the prognostic factors. In such analysis, it is crucial to demonstrate additional information gain from genetic markers. We provided ways to assess this gain both for an independent validation set and cross-validated test sets. Comparison of the selected genes between without and with adjustment for prognostic factors would provide some insights in understanding biological mechanisms in the disease progression and help determine a set of genes for further investigation in subsequent studies. It is advisable that the comparison is supplemented by analyses of differentially expressed genes across different levels of prognostic factors.

\section{Conclusion}

We develop a methodology for predicting survival outcomes using a subset of significant genes in prognostic marker studies with microarrays. The adequacy of the methodology was demonstrated through its application

Table 2: The selected genes from the gene filtering with no adjustment for IPI Score and from that with the adjustment by geneexpression signature.

\begin{tabular}{|c|c|c|c|c|c|c|c|}
\hline \multirow[t]{2}{*}{ Group } & \multicolumn{2}{|c|}{ Gene filtering } & \multicolumn{5}{|c|}{ Gene class } \\
\hline & No Adjust. & Adjust. & MHC-II & Proliferation & Lymph-node & Unknown & Total \\
\hline I & Selected & Selected & 16 & 20 & 1 & 6 & 43 \\
\hline II & Selected & Not Selected & 1 & 20 & 0 & 11 & 32 \\
\hline III & Not Selected & Selected & 7 & 23 & 0 & 12 & 42 \\
\hline
\end{tabular}


All patients

(A)

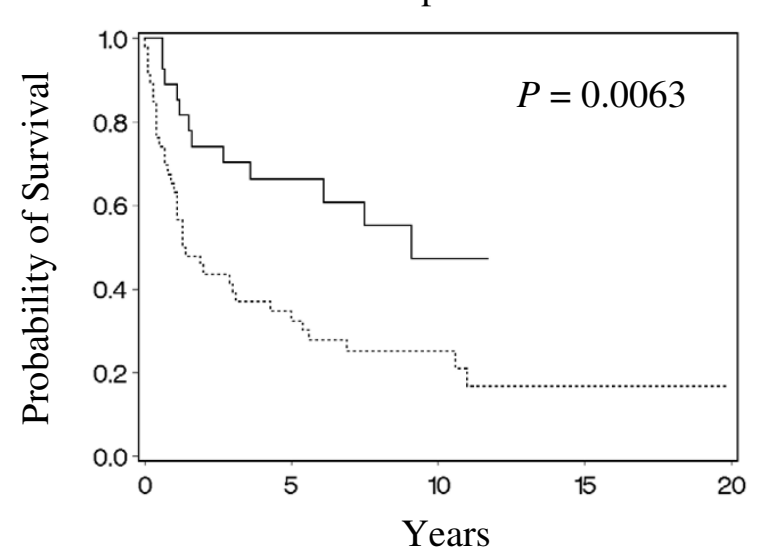

Low IPI risk stratum

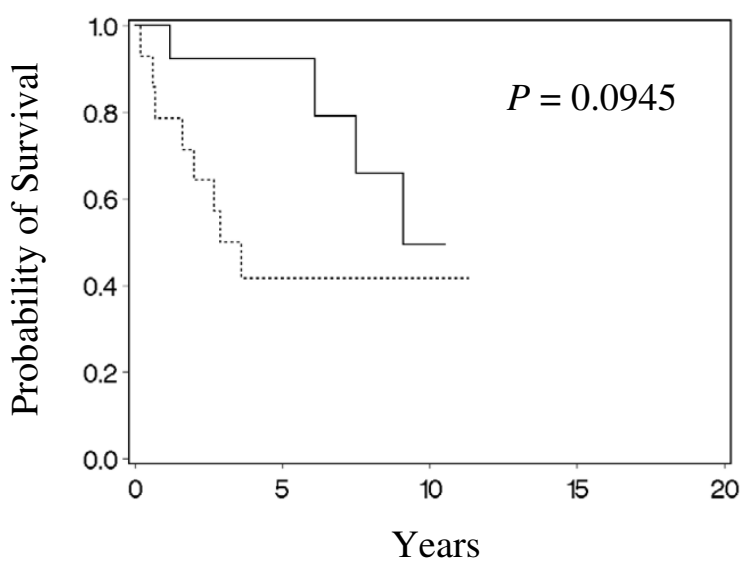

High IPI risk stratum

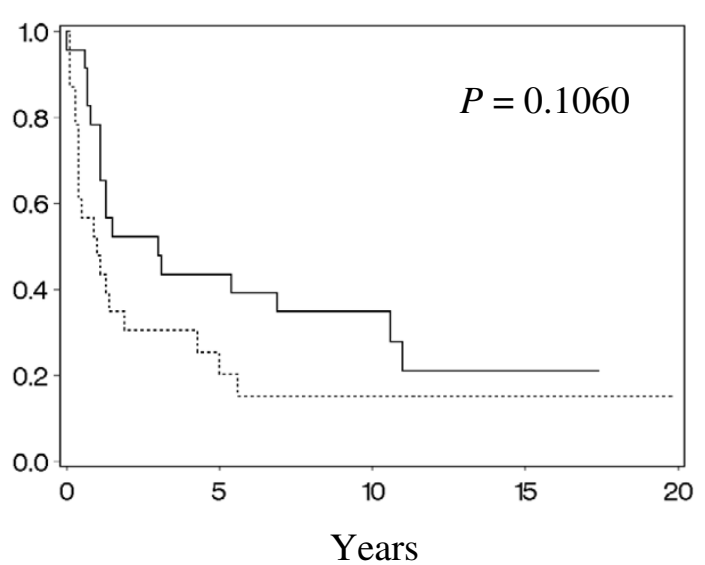

\section{Figure 5}

Kaplan-Meier estimates of survival for the two groups based on the prognostic index (the low prognostic index group (solid curves) and the high prognostic index group (dashed curves) for all patients (Panel A) and for each stratum by IPI Score (Panel B) in the validation set. The $P$-value in graph pertains to a two-sided log-rank test. The $P$ value for a stratified log-rank test is 0.0293 .

to simulated and real data. Our methodology can provide new insights based on predictive capability, possibly incorporating standard prognostic factors, in selecting a fraction of relevant genes for subsequent studies.

\section{Acknowledgements}

We would thank Seiichiro Yamamoto of National Cancer Center Research Institute, Takeharu Yamanaka of Translational Research Informatics Center, and Richard Simon of Biometric Research Branch, National Cancer Institute for helpful discussions on an earlier draft of this article.

\section{References}

I. Rosenwald A, Wright G, Chan WC, Connors JM, Campo E, Fisher RI, et al:: The use of molecular profiling to predict survival after chemotherapy for diffuse large-B-cell lymphoma. N Eng J Med 2002, 346: 1937-1947.

2. Dave SS, Wright G, Tan B, Rosenwald A, Gascoyne RD, Chan WC, Fisher RI, et al.: Prediction of survival in follicular lymphoma based on molecular features of tumor-infiltrating immune cells. N Eng J Med 2004, 35 I:2 I59-2 I69.

3. Bullinger L, Dohner K, Bair E, Frohling S, Schlenk RF, Tibshirani R, Dohner H, Pollack JR: Use of gene-expression profiling to identify prognostic subclasses in adult acute myeloid leukemia. $\mathrm{N}$ Eng J Med 2004, 350:1605-1616.

4. Beer DG, Kardia SLR, Huang CC, Giordano T], Levin AM, Misek DE, et al:: Gene-expression profiles predict survival of patients with lung adenocarcinoma. Nature Medicine 2002, 8:816-824.

5. Vasselli JR, Shih JH, lyengar SR, Maranchie J, Riss J, Worrell R, et al.: Predicting survival in patients with metastatic kidney cancer by gene-expression profiling in the primary tumor. Proc Nat Acad Sci USA 2003, 100:6958-6963.

6. Lossos IS, Czerwinski DK, Alizadeh AA, Wechser MA, Tibshirani R, Botstein D, Levy R: Prediction of survival in diffuse large-B-cell lymphoma based on the expression of six genes. N Eng J Med 2004, 350: 1828-1837.

7. Paik S, Shak S, Tang G, Kim C, Baker J, Cronin M, et al.: A multigene assay to predict recurrence of tamoxifen-treated, node-negative breast cancer. N Eng J Med 2004, 35 I:2817-2826. 
8. Nguyen DV, Rocke DM: Partial least squares proportional hazard regression for application to DNA microarray survival data. Bioinformatics 2002, 18:1625-1632.

9. Park PJ, Tian L, Kohane IS: Linking gene expression data with patient survival times using partial least squares. Bioinformatics 2002, I8(SuppI I):SI20-127.

10. Li H, Gui J: Partial Cox regression analysis for high-dimensional microarray gene expression data. Bioinformatics 2004, 20(Suppl I): i208-i2 I5

II. Hastie T, Tibshirani R: Efficient quadratic regularization for expression arrays. Biostatistics 2004, 5:329-340.

12. Pawitan $Y$, Bjohle J, Wedren S, Humphreys K, Skoog L, Huang F, Amler L, Shaw P, Hall P, Bergh J: Gene expression profiling for prognosis using Cox regression. Stat Med 2004, I 5: I 767- 1780.

13. Tukey JW: Tightening the clinical trial. Controlled Clin Trials 1993 , 14:266-285

14. Radmacher MD, McShane LM, Simon R: A paradigm for class prediction using gene expression profiles. J Comp Biol 2002, 9:505-5II.

15. Korn E, Simon R: Measures of explained variation for survival data. Stat Med 1990, 9:487-503.

16. Schemper M, Henderson R: Predictive accuracy and explained variation in Cox regression. Biometrics 2000, 56:249-255.

17. O'Quigley J, Xu R: Explained variation in proportional hazards regression. In Handbook of statistics in Clinical Oncology 2nd edition. Edited by: Crowley JJ. Ankerst DA, Boca Raton: Chapman \& Hall/CRC Press; 2006; 347-363; 2001:397-409.

18. Verweij M, Houwelingen V: Cross-validation in survival analysis. Stat Med I 993, I 2:2305-23 |4.

19. Pauler DK, Hardin J, Faulkner JR, LeBlanc M, Crowley JJ: Survival analysis with gene expression arrays. In Handbook of Statistics, Advances in Survival Analysis Volume 23. Edited by: Balakrishnan N, Rao CR. Amsterdam: Elsevier; 2004:675-688.

20. Simon R, Altman DG: Statistical aspects of prognostic factor studies in oncology. Br J Cancer 1994, 69:979-985.

21. Kalbfleisch JD, Prentice RL: The Statistical Analysis of Failure Time Data 2nd edition. Wiley; 2002.

22. Hsieh FY, Lavori PW: Sample-size calculations for the Cox proportional hazards regression model with nonbinary covariates. Controlled Clin Trials 2000, $21: 552-560$.

23. Molinaro AM, Simon R, Pfeiffer RM: Prediction error estimation: a comparison of resampling methods. Bioinformatics 2005, 21:330I-3307.

24. Ambroise C, McLachlan GJ: Selection bias in gene extraction on the basis of microarray gene-expression data. Proc Nat Acad Sci USA 2002, 99:6562-6566.

25. Simon R, Radmacher MD, Dobbin K, McShane LM: Pitfalls in the use of DNA microarray data for diagnostic and prognostic classification. J Nat Cancer Inst 2003, 95: I4-I8.

26. Matsui S: Statistical Applications using DNA microarrays for cancer diagnosis and prognosis. In Handbook of statistics in Clinical Oncology 2nd edition. Edited by: Crowley JJ, Ankerst DA. Boca Raton: Chapman and Hall/CRC Press :419-436.

27. The International Non-Hodgkin's Lymphoma Prognostic Factors Project: A predictive model for aggressive non-Hodgkin's lymphoma. N Engl J Med 1993, 329:987-994.

28. Bair E, Tibshirani R: Semi-supervised methods to predict patient survival from gene expression data. PLoS Biology 2004, 2:05II-0522.

29. Little RJA, Rubin DB: Statistical Analysis with Missing Data 2nd edition. Wiley; 200I.

30. Heagerty PJ, Lumley T, Pepe MS: Time-dependent ROC curves for censored survival data and a diagnostic marker. Biometrics 2000, 56:337-344.
Publish with Bio Med Central and every scientist can read your work free of charge

"BioMed Central will be the most significant development for disseminating the results of biomedical research in our lifetime. "

Sir Paul Nurse, Cancer Research UK

Your research papers will be:

- available free of charge to the entire biomedical community

- peer reviewed and published immediately upon acceptance

- cited in PubMed and archived on PubMed Central

- yours - you keep the copyright

Submit your manuscript here:

http://www.biomedcentral.com/info/publishing_adv.asp
BioMedcentral 